\title{
EDITORIAL
}

\section{SAFETY FIRST}

\author{
With a potential influenza pandemic looming, how practical is it to conduct vital experiments on \\ emergent and often lethal pathogens?
}

A recent News feature and Commentary published in Nature on the spectre of an influenza pandemic threw into sharp focus the potentially imminent threat of the emergence of a new and deadly influenza-virus strain. However, influenza is not the only viral threat to human health. SARS, West Nile virus and the haemorrhagic fever viruses are just a few examples of emerging and reemerging pathogens that cause potentially fatal human infections. Lest we forget, there are also continuing bioterror concerns over the potential deliberate deployment of disease-causing microorganisms.

Naturally, research on these pathogens and the development of therapeutics and vaccines to tackle them are a priority. But what are the practicalities for researchers carrying out experiments on such dangerous organisms?

Although different countries have different safety guidelines for research into microbial pathogens there is a consensus that containment is an absolute must for the most dangerous pathogens. Exotic viral pathogens are usually designated biosafety level 4 (BSL-4), the most stringent set of containment conditions. A new pandemic influenza strain would likely be classified as BSL-3 to BSL-4.

The emergence of new pathogens and an increased awareness of bioterrorism has ignited a containmentlaboratory building boom worldwide. Not only are these facilities expensive - the new BSL-4 lab at the University of Texas Medical Branch (UTMB) cost an estimated US $\$ 18$ million - they must be constructed by specialist companies to ensure that rigorous standards are met. The US currently has 6 BSL- 4 labs, 4 of which are military.There are a handful of BSL- 4 facilities in Europe, and although Japan has BSL-4 facilities, they are largely unused owing to opposition from local residents.

Wherever their location it is clear that not all BSL-4 facilities are created equal. Some facilities are simply glove-box isolators in which samples are accessed through glove-enclosed portals. Others are fully self-contained labs with airlocks that are accessed by researchers wearing pressure suits. Two important issues associated with BSL-4 laboratories are their availability - the cost of construction and maintenance - and whether they can support the experiments that scientists need to conduct. There are few large-animal facilities, even though these are essential to study zoonotic viruses.

Currently there are no international standards of accreditation for those working in BSL-4 facilities, although there are rigorous and intensive training programmes available. At the UTMB BSL-4 facility researchers must complete a 6-month training programme Although it is feasible to collaborate and use BSL-4 facilities, visiting scientists must be chaperoned in the BSL-4 laboratory or face months of training. A related issue is the question of whether there are sufficient trained personnel to work in all the available BSL-4 laboratories, and the costs associated with meeting their training needs. Containment laboratories need trained researchers and technicians, as well as administrators and safety officers that can deal with the increasingly difficult problems of transporting, storing and documenting research on dangerous pathogens.

A final issue facing researchers toiling against nature's deadliest pathogens is one of isolation - not of the pathogens but of the researchers themselves. Although collaboration is vital for research, scientists that focus on deadly pathogens often find themselves isolated in every sense of the word. BSL- 4 facilities typically house either animal-virus research or human-virus research, two fields with obvious collaborative potential. Yet only the BSL-4 lab in Winnipeg, Canada, houses both types of research.

To fight emerging and re-emerging microbial pathogens it is clear that containment facilities are urgently required. Perhaps the biggest impediment to progress is the lack of a financial commitment but the cost of building these laboratories is only the tip of the iceberg. The maintenance costs of these facilities also carries a heavy financial burden. A global commitment to provide adequate infrastructure to study highly infectious pathogens is essential. Overlooking this responsibility will undoubtedly impede progress, not only in understanding these infectious agents but, ultimately, in defeating them. 